\title{
RECIPROCAL RELATIONS, BOUNDS, AND SIZE EFFECTS FOR COMPOSITES WITH HIGHLY CONDUCTING INTERFACE*
}

\author{
ROBERT LIPTON ${ }^{\dagger}$
}

\begin{abstract}
We provide a reciprocal relation linking the effective conductivity of a composite with highly conducting phase interfaces to that of a composite with the same phase geometry but with an electrical contact resistance at phase interfaces. A field relationship linking the electric field inside a composite with highly conducting phase interfaces to the current in a composite with contact resistance between phases is found. New size effects exhibited by isotropic particulate suspensions with highly conducting interface are obtained. The effective properties of periodic composites are shown to be monotonically increasing as the size of the period cell tends to zero. The role of surface energy for energy minimizing polydisperse suspensions of disks is examined; a necessary condition for isotropic polydisperse suspensions with minimal effective conductivity is found. For monodisperse suspensions of spheres, a critical radius is found for which the electric field is uniform throughout the composite.
\end{abstract}

Key words. composite medium, contact resistance, reciprocal relation

AMS subject classifications. 35B27, 35J20, 78A30, 73B27

PII. S0036139995291180

1. Introduction. A highly conducting interface may be thought of as the limiting case of electrical transport across bulk phases separated by a thin highly conducting interphase layer. To a first approximation, the highly conducting interface is characterized by a discontinuous current field across the interface. The jump in the normal current produces an interfacial charge density. The associated electric potential is continuous across the interface and is coupled to the interfacial charge density through a Poisson equation on the interface; see (2.2) and (2.3). For a rigorous treatment we refer the reader to [13].

On the other hand, electrical contact resistance often appears due to the presence of a thin highly resistive layer or "interphase" between two conducting phases. The effects of the thin layer can also be modeled by an interface with suitable discontinuous transmission conditions. Here the electric potential jumps across the interface. The associated current normal to the interface is continuous and is proportional to the jump in electric potential. This is rigorously established in [14]. Both of these transmission conditions are distinct from the standard "perfectly bonded interface conditions," where both electric potential and normal current are continuous across the interface. We remark that contact resistance is not limited to electrostatic problems and can appear in the mathematically analogous context of heat conductivity. Here contact resistance can arise due to surface roughness [6] or to acoustic mismatch between phases at liquid helium temperatures; see [3].

The effective electrical conductivity for two-phase composites with highly conducting interfaces is investigated in the context of a periodic two-dimensional, twophase medium. The distribution of phases within the period cell can be arbitrary. The composite may be regarded as consisting of parallel cylinders of conductivity $\sigma_{1}$ and $\sigma_{2}$. We suppose that the highly conducting interface is characterized by a

\footnotetext{
* Received by the editors September 5, 1995; accepted for publication (in revised form) March 13, 1996. This research was supported in part by NSF grant DMS 9403866.

http://www.siam.org/journals/siap/57-2/29118.html

†Worcester Polytechnic Institute, Worcester, MA 01609 (lipton@wpi.edu).
} 
constant scalar tangential conductivity $\alpha$. We provide a reciprocal relation linking the effective conductivity of a composite with highly conducting interfaces to that of a composite with the same geometry but with electrical contact resistance at the two-phase interface; see Theorem 3.1. This result is shown to hold in general, with no symmetry assumptions on the composite geometry.

For the classical case of perfect contact between phases, we recover the well-known phase interchange relation proposed and proven by Keller (in 1964) for composites with rectangular symmetry; see [4]. More generally, we recover the interchange result of Mendelson (in 1975) for composites with diagonal effective tensors; see [10].

For a fixed geometry, we identify the relationship linking the electric field in a composite with highly conducting interfaces to the current field in a composite with interfacial contact resistance. (See Theorem 3.5.) These fields are seen to be related by a 90 -degree rotation.

More generally, we consider any periodic arrangement of two conductors in three dimensions with highly conducting interface. We exhibit a size effect for the effective conductivity tensor under rescaling. (See section 4.) It is seen that the effective property is monotone increasing as the scale of the period cell tends to zero. A related phenomenon was found for composites with interfacial contact resistance in Lipton [7]. This is in sharp contrast to the scale invariance enjoyed by composites with perfect contact between phases.

Recently, in Lipton and Vernescu [8], new upper and lower bounds on the effective conductivity for two-phase conductors with contact resistance were obtained. We use the reciprocal relation proposed here together with these results to obtain new bounds on the effective conductivity tensor for isotropic two-dimensional, two-phase, particulate composites with highly conducting interface. The lower bounds are found to depend upon component area fractions and geometric parameters of the interface. The upper bound is given in terms of the tortuosity of the connected matrix phase and the specific interfacial arclength. (See Theorem 5.1.) The upper bound is shown to be optimal in the limit $\alpha=\infty$; see Remark 5.3.

The monotonicity of the bounds in the interfacial geometric parameters and specific arclength is used to predict new size effects for the effective tensor. We consider suspensions of inclusions of conductivity $\sigma_{1}$ embedded in a matrix of higher conductivity $\sigma_{2}$. A distinguished parameter $P_{c r}=\alpha /\left(\sigma_{2}-\sigma_{1}\right)$ is found. This parameter measures the relative importance of the tangential conductivity to the contrast between phase conductivities. For monodisperse suspensions of disks this parameter gives the critical radius for which the effective conductivity equals that of the matrix; see Theorem 6.1. For radii below this value the effective conductivity surpasses that of the matrix, see Theorem 6.1. Physically, all size effects are due to the increase in the fraction ratio of specific interfacial arclength to particle area as the sizes of the inclusions decrease. The parameter $P_{c r}$ picks out the scale at which the effects of the interface balance the mismatch between the conductivities of each phase.

The aforementioned results can be extended to isotropic polydisperse suspensions of disks and for inclusions of any shape and distribution; see Theorems 6.2 and 6.3. It is shown that the effective conductivity always lies above that of the matrix, provided that the mean radius of the polydisperse suspension lies below $P_{c r}$. More generally for isotropic suspensions of particles of any shape and distribution we find that the effective conductivity lies below that of the matrix when the specific arclength-toparticle-area fraction lies below $2 P_{c r}{ }^{-1}$. We apply these theorems to address the role of surface energy when designing energy minimizing arrangements of inclusions; see 
Theorem 6.4. Here we fix the area fraction and find a necessary condition for the isotropic polydisperse suspension with minimal effective conductivity.

We note that for isotropic monodisperse suspensions of disks the critical radius is directly related to the notion of a critical value for the dimensionless tangential conductivity studied in [15]. We show that this critical phenomenon persists even for anisotropic suspensions of disks and (spheres) in arbitrary domains in two and (three) dimensions; see Corollary 7.2 and Remark 7.3. In doing so we obtain a fundamental result concerning the behavior of electric fields inside suspensions at critical radius; see Theorem 7.1. We find that the electric field is uniform throughout the composite for suspensions of disks (spheres) at critical radius in two and (three) dimensions.

Based upon the results of Theorem 7.1, we address the following design problem in three dimensions. We consider an arbitrary region $\Omega$ filled with a monodisperse suspension of spheres of conductivity $\sigma_{1}$ in a matrix of conductivity $\sigma_{2}$. Here $\sigma_{2}>\sigma_{1}$, and we prescribe the common radii of the spheres. We show how to choose a highly conducing interface with the appropriate tangential conductivity $\alpha$ that renders the spheres undetectable when the boundary of $\Omega$ is subjected to any uniform current; see Corollary 7.4 and Remark 7.6. Indeed, we show that for the proper choice of coating, the resulting electrical field is the same as the electric field that would occur in the absence of the spheres. Moreover, the resulting current external to the spheres is unaffected by their presence.

Last, we note that although we have used the terminology of electrical conductivity, our results apply equally to the contexts of thermal conductivity, magnetic permeability, and diffusivity.

2. Effective conductivity for composites with highly conducting interface. We consider a unit square $Q$ filled with two isotropic conductors with conductivities specified by $\sigma_{1}$ and $\sigma_{2}$. In what follows we make no assumption on the distribution of the conductors within the interior of the domain. One can think of the cube as representing a (possibly very complicated) period cell for a composite material. Decomposing the electric potential into a periodic fluctuation $\tilde{\varphi}$ and a linear part $\mathbf{E} \cdot x$ the average electric field inside $Q$ is

$$
\mathbf{E}=\int_{\partial Q}(\tilde{\varphi}+\mathbf{E} \cdot x) \mathbf{n} d s
$$

Here $\partial Q$ is the boundary of the cube and $\mathbf{n}$ is the outer normal to the boundary. To fix ideas we assume that the two-phase boundary is sufficiently smooth (i.e., a twice differentiable curve). The fluctuating part of the potential is continuous across phase interfaces and satisfies

$$
\Delta \tilde{\varphi}=0 \quad \text { inside each phase }
$$

and

$$
\sigma_{1}(\nabla \tilde{\varphi}+\mathbf{E})_{1} \cdot \mathbf{n}-\sigma_{2}(\nabla \tilde{\varphi}+\mathbf{E})_{2} \cdot \mathbf{n}=\alpha \Delta(\tilde{\varphi}+\mathbf{E} \cdot x)
$$

on the phase boundary $\Gamma$. Subscripts 1 and 2 denote the side of the interface where field quantities are evaluated. Here $\mathbf{n}$ is the unit normal pointing into phase 2, and $\Delta$ is the Laplace-Beltrami operator on $\Gamma$ defined by

$$
\Delta(\tilde{\varphi}+\mathbf{E} \cdot x)=\delta_{i} \delta_{i}(\tilde{\varphi}+\mathbf{E} \cdot x)
$$


where $\delta$ is the tangential gradient of $\tilde{\varphi}+\mathbf{E} \cdot x$ on $\Gamma$, i.e.,

$$
\delta_{i}(\tilde{\varphi}+\mathbf{E} \cdot x)=\partial_{x_{i}}(\tilde{\varphi}+\mathbf{E} \cdot x)-(\mathbf{n} \cdot(\nabla \tilde{\varphi}+\mathbf{E})) \mathbf{n}_{i} .
$$

We observe from (2.3) that the current suffers a discontinuity at the two-phase interface. The jump in current provides a surface charge density which drives the Poisson equation (2.3) on the two-phase interface. The highly conducting interface may be thought of as the limiting case of electric transport across two phases separated by a thin highly conducting interphase layer. We denote the conductivity of the interphase layer by $\sigma_{i}$ and its thickness by $l$. Here the tangential conductivity $\alpha$ is the finite limit of the product $l \sigma_{i}$ as $\sigma_{i}$ tends to infinity and $l$ tends to zero; see [13].

Denoting the local conductivity by $\sigma(x)$, the (possibly anisotropic) effective conductivity tensor $\sigma_{+}{ }^{e}$ of the mixture as measured by an outside observer is defined as

$$
\sigma_{+}{ }^{e} \mathbf{E}=\int_{\partial Q} \sigma(x)(\nabla \tilde{\varphi}+\mathbf{E}) \cdot \mathbf{n} x d S .
$$

Integration by parts and application of (2.2), (2.3), and the natural boundary condition for the current yield

$$
\sigma_{+}{ }^{e} \mathbf{E} \cdot \mathbf{E}=\int_{Q} \sigma(x)|\nabla \tilde{\varphi}+\mathbf{E}|^{2} d x+\alpha \int_{\Gamma}|\delta(\tilde{\varphi}+\mathbf{E} \cdot x)|^{2} d s .
$$

Physically (2.7) is a relation between the total energy dissipation rate inside the heterogeneous conductor and the energy dissipated in a homogeneous effective conductor.

One easily verifies the Dirichlet-like variational principle for the effective conductivity:

$$
\sigma_{+}{ }^{e} \mathbf{E} \cdot \mathbf{E}=\min _{\varphi \in V} \int_{Q} \sigma(x)|\nabla \varphi+\mathbf{E}|^{2} d x+\alpha \int_{\Gamma}|\delta(\varphi+\mathbf{E} \cdot x)|^{2} d s,
$$

where the space of trial fields is given by

$$
V=\left\{\varphi \in W^{1,2}(Q) \mid \varphi-Q \text { periodic }\right\} .
$$

For completeness we provide the field equations and definition of effective conductivity for composites with interfacial barrier resistance.

We consider the same composite with phases of conductivity $c_{1}$ and $c_{2}$ with interfacial contact resistance specified by $\beta^{-1}$. Here $\beta$ may be regarded as the interfacial barrier conductance. As before the electric potential is decomposed into a periodic fluctuation $\tilde{\varphi}$ and a linear part. The average field, measured by an outside observer, is

$$
\zeta=\int_{\partial Q}\{\tilde{\psi}+\zeta \cdot x\} \mathbf{n} d s .
$$

The fluctuating part of the potential satisfies

$$
\Delta \tilde{\psi}=0 \quad \text { inside each phase }
$$

and

$$
\begin{gathered}
c_{1}(\nabla \tilde{\psi}+\zeta)_{1} \cdot \mathbf{n}=c_{2}(\nabla \tilde{\psi}+\zeta)_{2} \cdot \mathbf{n}, \\
c_{2}(\nabla \tilde{\psi}+\zeta)_{2} \cdot \mathbf{n}=-\beta\left(\tilde{\psi}_{1}-\tilde{\psi}_{2}\right)
\end{gathered}
$$


on the two-phase interface. Condition (2.13) accounts for the interfacial contact resistance. Here the jump in potential is proportional to the current passing across the interface. The local conductivity is denoted by $c(x)$, and the effective conductivity of the mixture $\sigma_{-}{ }^{e}$ is given by

$$
\sigma_{-}{ }^{e} \zeta=\int_{\partial Q} c(x)(\nabla \tilde{\psi}+\zeta) \cdot \mathbf{n} x d s .
$$

For our work we will use a Thompson-like variational principle describing the effective conductivity; see Lipton and Vernescu [8]. The effective conductivity is given by

$$
\left(\sigma_{-}{ }^{e}\right)^{-1} \overline{\mathbf{j}} \cdot \overline{\mathbf{j}}=\min _{j \in W} \int_{Q} c^{-1}(x)|j+\overline{\mathbf{j}}|^{2}+\beta^{-1} \int_{\Gamma}|(j+\overline{\mathbf{j}}) \cdot \mathbf{n}|^{2} d s .
$$

The space of trial fields is given by

$$
W=\left\{j \in L^{2}(Q)^{2} \mid \nabla \cdot j=0, \int_{Q} j d x=0, j \text { is } Q \text { periodic }\right\} .
$$

Last we remark that the field equations for two-phase "perfectly bonded" composites are given by

$$
\begin{gathered}
\Delta \psi=0 \quad \text { in each phase, } \\
\sigma_{1}(\nabla \psi+\zeta)_{1} \mathbf{n}=\sigma_{2}(\nabla \psi+\zeta)_{2} \cdot \mathbf{n},
\end{gathered}
$$

and the fluctuating potential $\psi$ is continuous across the phase interface. The associated effective conductivity is denoted by $\sigma_{p}{ }^{e}$ and defined by

$$
\sigma_{p}^{e} \zeta=\int_{\partial Q} \sigma(x)(\nabla \psi+\zeta) \cdot \mathbf{n} x d s .
$$

REMARK 2.1. For composites with highly conducting interface we remark that in the $\alpha=0$ limit the effective conductivity reduces to the effective conductivity of a perfectly bonded composite. Similarly, the effective conductivity of a composite with interfacial contact resistance agrees with that of a perfectly bonded composite in the $\beta=\infty$ limit.

3. Reciprocal relations, phase interchange theorems, and field relations. We partition the unit square $Q$ into three sets, $\mathbf{Y}_{a}, \mathbf{Y}_{b}$, and $\Gamma$. Here $\Gamma$ denotes the two-phase interface, and the regions $\mathbf{Y}_{a}$ and $\mathbf{Y}_{b}$ can be filled with either conductor $\sigma_{1}$ or $\sigma_{2}$. For a fixed two-phase geometry the effective conductivity tensor may be regarded as a matrix-valued function of its component conductivities. We consider the effective conductivity for a composite with highly conducting interface and write $\sigma_{+}{ }^{e}=\left(\sigma_{1}, \sigma_{2}, \alpha\right)$, where the first argument represents the conductivity in $\mathbf{Y}_{a}$, the second is the conductivity in $\mathbf{Y}_{b}$, and the third is the conductivity on the interface. One readily checks that this function is homogeneous of degree one in its arguments; i.e., for any scalar $t$

$$
\sigma_{+}{ }^{e}\left(t \sigma_{1}, t \sigma_{2}, t \alpha\right)=t \sigma_{+}{ }^{e}\left(\sigma_{1}, \sigma_{2}, \alpha\right) .
$$

The corresponding effective conductivity tensor of a composite with interfacial contact resistance is also homogeneous of degree one in its component conductivities $\sigma_{1}, \sigma_{2}$, 
and $\beta$, and we write $\sigma_{-}{ }^{e}=\sigma_{-}{ }^{e}\left(\sigma_{1}, \sigma_{2}, \beta\right)$. We introduce the matrix $\mathcal{R}$ associated with a counterclockwise rotation of $\pi / 2$ radians and state the following theorem.

THEOREM 3.1 (reciprocal relation).

$$
\sigma_{+}{ }^{e}\left(\sigma_{1}, \sigma_{2}, \alpha\right)=\mathcal{R}\left(\sigma_{-}{ }^{e}\left(\frac{1}{\sigma_{1}}, \frac{1}{\sigma_{2}}, \frac{1}{\alpha}\right)\right)^{-1} \mathcal{R}^{T} .
$$

It follows from Theorem 3.1 that the tensors $\sigma_{+}{ }^{e}\left(\sigma_{1}, \sigma_{2}, \alpha\right)$ and $\sigma_{-}{ }^{e}\left(\frac{1}{\sigma_{1}}, \frac{1}{\sigma_{2}}, \frac{1}{\alpha}\right)$ are simultaneously diagonalizable. Denoting the eigenvalues of $\sigma_{+}{ }^{e}$ and $\sigma_{-}{ }^{e}$ by $\left(\sigma_{+}{ }^{e}, \sigma_{+2}{ }^{e}\right)$ and $\left(\sigma_{-1}^{e}, \sigma_{-2}^{e}\right)$, respectively, we have the following corollary.

COROLlary 3.2.

$$
\sigma_{-2}^{e}\left(\frac{1}{\sigma_{1}}, \frac{1}{\sigma_{2}}, \frac{1}{\alpha}\right)=1 / \sigma_{+1}^{e}\left(\sigma_{1}, \sigma_{2}, \alpha\right)
$$

and

$$
\sigma_{-1}^{e}\left(\frac{1}{\sigma_{1}}, \frac{1}{\sigma_{2}}, \frac{1}{\alpha}\right)=1 / \sigma_{+2}^{e}\left(\sigma_{1}, \sigma_{2}, \alpha\right) .
$$

We make use of the homogeneity property of the functions ${\sigma_{+}}^{e}$ and $\sigma_{-}{ }^{e}$ to obtain the following phase interchange identity.

COROLLARY 3.3 (phase interchange identity for anisotropic composites).

$$
\sigma_{-2}^{e}\left(\sigma_{2}, \sigma_{1}, \frac{\sigma_{1} \sigma_{2}}{\alpha}\right) / \sigma_{2}=\sigma_{1} / \sigma_{+1}^{e}\left(\sigma_{1}, \sigma_{2}, \alpha\right)
$$

and

$$
\sigma_{-1}^{e}\left(\sigma_{2}, \sigma_{1}, \frac{\sigma_{1} \sigma_{2}}{\alpha}\right) / \sigma_{2}=\sigma_{1} / \sigma_{+2}^{e}\left(\sigma_{1}, \sigma_{2}, \alpha\right) .
$$

When the composite is isotropic, the effective conductivity is a scalar-valued function, and from Corollary 3.3 we obtain the following corollary.

COROLLARY 3.4 (phase interchange identity for isotropic composites).

$$
\sigma_{-}{ }^{e}\left(\sigma_{2}, \sigma_{1}, \frac{\sigma_{1} \sigma_{2}}{\alpha}\right) \sigma_{+}{ }^{e}\left(\sigma_{1}, \sigma_{2}, \alpha\right)=\sigma_{2} \sigma_{2} .
$$

We now show how to recover Keller's (1964) and Mendelson's (1975) phase interchange result in the limit as the tangential conductivity tends to zero. The effective conductivity function of a perfectly bonded composite depends upon the bulk conductivities only. Thus, for a composite of conductivity $c_{1}$ in $\mathbf{Y}_{a}$ and $c_{2}$ in $\mathbf{Y}_{b}$ we write $\sigma_{p}{ }^{e}=\sigma_{p}{ }^{e}\left(c_{1}, c_{2}\right)$. From Remark 2.1, it follows that

$$
\sigma_{+}{ }^{e}\left(c_{1}, c_{2}, 0\right)=\sigma_{-}{ }^{e}\left(c_{1}, c_{2}, \infty\right)=\sigma_{p}{ }^{e}\left(c_{1}, c_{2}\right) .
$$

Passing to the $\alpha=0$ limit in Corollary 3.3 and applying (3.8), we recover the following phase interchange relation:

$$
{\sigma_{p_{2}}}^{e}\left(\sigma_{2}, \sigma_{1}\right) / \sigma_{2}=\sigma_{1} / \sigma_{p_{1}}{ }^{e}\left(\sigma_{1}, \sigma_{2}\right) .
$$

Equation (3.9) is precisely Keller's result [4] when the composite possesses rectangular symmetry. When the effective conductivity is diagonal, (3.9) is the relation pointed out by Mendelson [10]. For a partition of $Q$ into the sets $\mathbf{Y}_{a}, \mathbf{Y}_{b}$, and $\Gamma$, 
we denote as before the electric field $\nabla \tilde{\varphi}+\mathbf{E}$ for a composite conductor with highly conducting interface with conductivity $\sigma_{1}$ in $\mathbf{Y}_{a}, \sigma_{2}$ in $\mathbf{Y}_{b}$, and tangential conductivity $\alpha$ on $\Gamma$. We denote by $\tilde{\mathbf{j}}$ the current in a composite conductor with interfacial contact resistance with conductivity $\sigma_{1}{ }^{-1}$ in $\mathbf{Y}_{a}, \sigma_{2}{ }^{-1}$ in $\mathbf{Y}_{b}$, and interfacial barrier conductance $\alpha^{-1}$ on $\Gamma$. We show that these fields are related by a counterclockwise rotation of $\pi / 2$ radians.

THEOREM 3.5.

$$
\nabla \tilde{\varphi}+\mathbf{E}=\mathcal{R} \tilde{\mathbf{j}} \quad \text { in } \mathbf{Y}_{a}
$$

and

$$
\nabla \tilde{\varphi}+\mathbf{E}=\mathcal{R} \tilde{\mathbf{j}} \quad \text { in } \mathbf{Y}_{b}
$$

where

$$
\tilde{\mathbf{j}}=\sigma_{1}^{-1}(\nabla \tilde{\psi}+\zeta) \quad \text { in } \mathbf{Y}_{a}
$$

and

$$
\tilde{\mathbf{j}}=\sigma_{2}{ }^{-1}(\nabla \tilde{\psi}+\zeta) \quad \text { in } \mathbf{Y}_{b} .
$$

Here the fluctuating field $\tilde{\psi}$ is a solution of the field equations (2.11)-(2.13) with $\zeta=\mathcal{R}^{T} \sigma_{+}{ }^{e} \mathbf{E}, c_{1}=\sigma_{1}{ }^{-1}, c_{2}=\sigma_{2}{ }^{-1}$, and $\beta=\alpha^{-1}$.

We conclude this section with proofs of Theorems 3.1 and 3.5.

Proof of Theorem 3.1. For a fixed partition $\mathbf{Y}_{a} \cup \mathbf{Y}_{b} \cup \Gamma=Q$, we consider a composite with interfacial barrier resistance with conductivity $c_{1}$ in $\mathbf{Y}_{a}, c_{2}$ in $\mathbf{Y}_{b}$, and barrier conductance $\beta$ on $\Gamma$. From the variational principle (2.15) we have for any constant current $\overline{\mathbf{j}}$ the identity

$$
\left(\sigma_{-}{ }^{e}\left(c_{1}, c_{2}, \beta\right)\right)^{-1} \overline{\mathbf{j}} \cdot \overline{\mathbf{j}}=\min _{\mathbf{j} \in W} \int_{Q} c^{-1}(x)|\mathbf{j}+\overline{\mathbf{j}}|^{2}+\beta^{-1} \int_{\Gamma}|(\mathbf{j}+\overline{\mathbf{j}}) \cdot \mathbf{n}|^{2} d S .
$$

We observe that every field $\mathbf{j}$ in $W$ is representable by a $Q$-periodic stream function $\varphi$ in $W^{1,2}(Q)$, where

$$
\mathbf{j}=\mathcal{R}^{T} \nabla \varphi
$$

Substitution of (3.15) into (3.14) yields

$$
\left(\sigma_{-}{ }^{e}\left(c_{1}, c_{2}, \beta\right)\right)^{-1} \overline{\mathbf{j}} \cdot \overline{\mathbf{j}}=\min _{\varphi \in V} \int_{Q} c^{-1}(x)|\nabla \varphi+\mathcal{R} \overline{\mathbf{j}}|^{2}+\beta^{-1} \int_{\Gamma}|(\nabla \varphi+\mathcal{R} \overline{\mathbf{j}}) \cdot \mathbf{t}|^{2} d S
$$

where $\mathbf{t}$ is the unit tangent vector to the interface and $\mathbf{t}=\mathcal{R} \mathbf{n}$. Here $V$ is the class of trials given by (2.9). Next we observe that in two dimensions $\delta_{i} \varphi=(\nabla \varphi \cdot \mathbf{t}) \mathbf{t}_{i}$ and that the second term on the right-hand side of (3.16) can be written as

$$
\int_{\Gamma}|\delta(\varphi+\mathcal{R} \overline{\mathbf{j}} \cdot x)|^{2} d S
$$
obtain

Last, applying (3.17) and writing $\overline{\mathbf{j}}=\mathcal{R}^{T} \mathcal{R} \overline{\mathbf{j}}$ on the left-hand side of (3.16) we

$$
\begin{gathered}
\mathcal{R}\left(\sigma_{-}{ }^{e}\left(c_{1}, c_{2}, \beta\right)\right)^{-1} \mathcal{R}^{T} \mathcal{R} \overline{\mathbf{j}} \cdot \mathcal{R} \overline{\mathbf{j}} \\
=\min _{\varphi \in V} \int_{Q} c^{-1}(x)|\nabla \varphi+\mathcal{R} \overline{\mathbf{j}}|^{2}+\beta^{-1} \int_{\Gamma}|\delta(\varphi+\mathcal{R} \overline{\mathbf{j}} \cdot x)|^{2} d S
\end{gathered}
$$


Substitution of $\mathbf{E}=\mathcal{R} \overline{\mathbf{j}}$ into the variational principle (2.8) gives

$$
\sigma_{+}{ }^{e}\left(\sigma_{1}, \sigma_{2}, \alpha\right) \mathcal{R} \overline{\mathbf{j}} \cdot \mathcal{R} \overline{\mathbf{j}}=\min _{\varphi \in V} \int_{Q} \sigma(x)|\nabla \varphi+\mathcal{R} \overline{\mathbf{j}}|^{2} d \mathbf{r}+\alpha \int_{\Gamma}|\delta(\varphi+\mathcal{R} \overline{\mathbf{j}} \cdot x)|^{2} d S .
$$

Choosing $\beta=\alpha^{-1}, c_{1}=\sigma_{1}^{-1}, c_{2}=\sigma_{2}^{-1}$ in (3.18), it follows from (3.19) that

$$
\sigma_{+}{ }^{e}\left(\sigma_{1}, \sigma_{2}, \alpha\right)=\mathcal{R}\left(\sigma_{-}{ }^{e}\left(\frac{1}{\sigma_{1}}, \frac{1}{\sigma_{2}}, \frac{1}{\alpha}\right)\right)^{-1} \mathcal{R}^{T},
$$

and the theorem is proven.

Proof of Theorem 3.5. We suppose that $\tilde{\varphi}$ in $W^{1,2}(Q)$ is the periodic solution of the field equations given by (2.2) and (2.3), with conductivity $\sigma_{1}$ in $\mathbf{Y}_{a}, \sigma_{2}$ in $\mathbf{Y}_{b}$, and tangential conductivity $\alpha$ on $\Gamma$. We introduce the potential $\psi$ defined up to a constant in each phase by

$$
\nabla \tilde{\varphi}+\mathrm{E}=\mathcal{R} \sigma_{1}^{-1}(\nabla \psi+\zeta) \text { in } \mathbf{Y}_{a}
$$

and

$$
\nabla \tilde{\varphi}+\mathrm{E}=\mathcal{R} \sigma_{2}{ }^{-1}(\nabla \psi+\zeta) \text { in } \mathbf{Y}_{b} .
$$

Multiplying both sides of (3.21) and (3.22) by $\mathcal{R}^{T}$ and taking the divergence of both sides yield

$$
\Delta \psi=0 \quad \text { in } \mathbf{Y}_{a} \cup \mathbf{Y}_{b} .
$$

Since $\tilde{\varphi}$ lies in $W^{1,2}(Q)$ the jump in $\tilde{\varphi}$ across phase interfaces is zero and so

$$
[(\nabla \tilde{\varphi}+\mathrm{E}) \cdot \mathbf{t}]=0,
$$

where [.] denotes a jump in a quantity across $\Gamma$ and $\mathbf{t}=\mathcal{R} \mathbf{n}$ is the unit tangent to the interface. Applying (3.21) and (3.22) and taking traces we find that

$$
\sigma_{1}^{-1}(\nabla \psi+\zeta)_{1} \cdot \mathbf{n}-\sigma_{2}{ }^{-1}(\nabla \psi+\zeta)_{2} \cdot \mathbf{n}=[(\nabla \tilde{\varphi}+\mathrm{E}) \cdot \mathbf{t}]=0 .
$$

Applying (3.21) and (3.22) and taking traces in (2.3) yield

$$
-\left((\nabla \psi+\zeta)_{1} \cdot \mathbf{t}-(\nabla \psi+\zeta)_{2} \cdot \mathbf{t}\right)=\alpha \Delta(\tilde{\varphi}+\mathbf{E} \cdot x)
$$

on $\Gamma$.

We observe that on the interface $\Delta(\tilde{\varphi}+\mathbf{E} \cdot x)=\partial_{t}^{2}(\tilde{\varphi}+\mathbf{E} \cdot x)$, where $\partial_{t}$ is the usual tangential derivative. Integration of (3.26) along the interface yields

$$
-\left(\psi_{1}-\psi_{2}\right)=\alpha\left(\partial_{t} \tilde{\varphi}+\mathbf{E} \cdot \mathbf{t}\right)+K,
$$

where $K$ is a constant of integration.

Applying (3.22) and taking traces give

$$
-\alpha^{-1}\left(\psi_{1}-\psi_{2}\right)=\sigma_{2}^{-1}(\nabla \psi+\zeta)_{2} \cdot \mathbf{n}+K .
$$

Noting that the potential $\psi$ is defined up to a constant in each phase we choose $\psi$ such that $K=0$ in (3.28). Taking the volume average of $\nabla \tilde{\varphi}+\mathbf{E}$ and application of (3.12) and (3.13) show that $\zeta=\mathcal{R}^{T} \sigma_{+}{ }^{e} \mathbf{E}$, and we conclude that $\psi$ is a solution of (2.11)-(2.13) with $c_{1}=\sigma_{1}{ }^{-1}, c_{2}=\sigma_{2}{ }^{-1}, \beta=\alpha^{-1}$, and $\zeta=\mathcal{R}^{T} \sigma_{+}{ }^{e} \mathbf{E}$. The theorem follows from (3.21) and (3.22), noting that the current in the composite with interfacial barrier resistance is given by $\tilde{\mathbf{j}}=\sigma_{1}^{-1}(\nabla \psi+\zeta)$ in $\mathbf{Y}_{a}$ and $\tilde{\mathbf{j}}=\sigma_{2}{ }^{-1}(\nabla \psi+\zeta)$ in $\mathbf{Y}_{b}$. 
4. Rescaling and size effects. We consider rescaled versions of a given twophase geometry. It is shown that the effective property monotonically increases as the scale of the period tends to zero. We let $\ell$ be a positive integer and $\mu$ be a positive scalar. We consider a composite with tangential conductivity $\alpha=\mu \ell$ and local conductivity $\sigma(x)$ taking the values $\sigma_{1}$ and $\sigma_{2}$. We denote the associated effective conductivity and fluctuating potential by $\sigma_{+}{ }^{\ell}$ and $\tilde{\varphi}^{\ell}$. Hence $\sigma_{+}{ }^{\ell}$ is given by

$$
\sigma_{+}^{\ell} E \cdot E=\int_{Q} \sigma(x)\left|\nabla \tilde{\varphi}^{\ell}+E\right|^{2} d x+\mu \ell \int_{\Gamma}\left|\delta\left(\tilde{\varphi}^{\ell}+E \cdot x\right)\right|^{2} d S .
$$

REMARK 4.1. One observes from the variational formulation (2.8) that $\sigma_{+}{ }^{\ell}$ is monotone increasing in $\ell$ (in the sense of quadratic forms).

Next, we consider a composite with a rescaled local conductivity $\sigma^{\ell}(x)=\sigma(\ell x)$ and tangential conductance $\alpha=\mu$. We denote the associated effective conductivity tensor and fluctuating potential by ${\sigma_{+}}^{e, \ell}$ and $\hat{\varphi}^{\ell}$, respectively. The effective conductivity $\sigma_{+}{ }^{e, \ell}$ is given by

$$
\sigma_{+}{ }^{e, \ell} E \cdot E=\int_{Q} \sigma^{\ell}(x)\left|\nabla \hat{\varphi}^{\ell}+E\right|^{2} d x+\mu \int_{\Gamma^{\ell}}\left|\delta\left(\hat{\varphi}^{\ell}+E \cdot x\right)\right|^{2} d S .
$$

Here $\Gamma^{\ell}$ is the two-phase interface. One easily checks that the two potentials are related by

$$
\hat{\varphi}^{\ell}(x)=\ell^{-1} \tilde{\varphi}^{\ell}(\ell x) .
$$

Upon substitution of (4.3) into (4.2) and rescaling we obtain the following.

THEOREM 4.2 (size effect theorem). The effective conductivity of a composite with local conductivity $\sigma(x)$ and tangential conductivity $\mu \ell$ is identical to that of a $1 / \ell$ periodic composite with local conductivity $\sigma^{\ell}(x)=\sigma(\ell x)$ and tangential conductivity $\mu$, i.e.,

$$
\sigma_{+}^{e, \ell}=\sigma_{+}^{\ell} .
$$

Moreover, from Remark 4.1 it follows that the effective tensor $\sigma_{+}{ }^{e, \ell}$ increases monotonically as the scale of the period (given by $\ell^{-1}$ ) tends to zero.

Physically, this corresponds to the fact that the surface-to-volume ratio of the highly conducting interface increases as the scale of the period tends to zero.

REMARK 4.3. An identical proof shows that ${\sigma_{+}}^{e, \ell}=\sigma_{+}{ }^{\ell}$ for periodic threedimensional conductors, and one naturally has that ${\sigma_{+}}^{e, \ell}$ is monotone increasing as the scale of the period tends to zero.

5. Bounds on the effective conductivity tensor. The reciprocal relation provides a means of obtaining bounds on the effective conductivity tensor for composites with highly conducting interface in terms of bounds on the effective tensor for composites with interfacial contact resistance. In Lipton and Vernescu [8], upper and lower bounds on the effective conductivity for composites with interfacial contact resistance were obtained. To fix ideas in this section and in section 6 we will consider only suspensions for which the associated effective tensor is isotropic. Such suspensions include those possessing cubic symmetries; cf. Nye [12]. We show how to obtain new bounds for the effective conductivity of particulate composites with highly conducting interface.

We suppose that the disconnected region occupied by the particles is denoted by $\mathbf{Y}_{a}$ and the matrix region by $\mathbf{Y}_{b}$. We suppose that the conductivity of the particles 
is $\sigma_{1}$ and that of the matrix is $\sigma_{2}$, with $\sigma_{1}<\sigma_{2}$. For a tangential conductivity $\alpha$, the effective conductivity is written $\sigma_{+}{ }^{e}\left(\sigma_{1}, \sigma_{2}, \alpha\right)$. The effective conductivity for the same geometry but with interfacial barrier resistance $\alpha$ and particle and matrix conductivities $\sigma_{1}{ }^{-1}$ and $\sigma_{2}{ }^{-1}$, respectively, is written $\sigma_{-}{ }^{e}\left(\sigma_{1}{ }^{-1}, \sigma_{2}{ }^{-1}, \alpha^{-1}\right)$. We denote the specific interfacial arclength by $s$ and the particle and matrix area fractions by $\theta_{a}$ and $\theta_{b}$, respectively. The area fractions satisfy $\theta_{b}=1-\theta_{a}$. Appealing to the bounds (II 2.10) and (III 3.38) given in Lipton and Vernescu [8], we have

$$
L\left({\sigma_{1}}^{-1},{\sigma_{2}}^{-1}, \alpha^{-1}, \theta_{a}\right) \leq{\sigma_{-}}^{e}\left({\sigma_{1}}^{-1},{\sigma_{2}}^{-1}, \alpha^{-1}\right) \leq \mathcal{U}\left({\sigma_{1}}^{-1},{\sigma_{2}}^{-1}, \alpha^{-1}, \theta_{a}\right) .
$$

Here the lower bound is given by

$$
L\left(\sigma_{1}^{-1}, \sigma_{2}{ }^{-1}, \alpha^{-1}, m, \theta_{a}\right)=\sigma_{2}^{-1}-\sigma_{2}^{-1}\left((1-m)^{-1}+\left(\sigma_{2}{ }^{-1} \theta_{a} c\right)^{-1}\right)^{-1},
$$

where $c=\frac{s \alpha}{2 \theta_{a}}-\left(\sigma_{2}-\sigma_{1}\right)$ and $m$ is the effective conductivity of the connected matrix phase filled with material of unit conductivity and particles filled with perfect insulators. We remark that the quantity $m^{-1}$ is often referred to in the porous media literature as the formation factor (cf. Dullien [2]). A second often-used parameter is the electrical tortuosity. This parameter can be obtained from nuclear magnetic resonance measurements. Roughly speaking the electrical tortuosity measures the effective average path length in a porous media, taking into account the effect of the constriction between inclusions. The formation factor is related to the electrical tortuosity $\tau$ of the matrix phase by

$$
\tau=\frac{\theta_{b}}{m}
$$

The upper bound on $\sigma_{-}{ }^{e}$ is given by

$$
\mathcal{U}\left(\sigma_{1}{ }^{-1}, \sigma_{2}{ }^{-1}, \alpha^{-1}, k, \theta_{a}\right)=\left(\sigma_{1}+\frac{\theta_{b} k /(2 \alpha)+\theta_{a}^{2} \lambda+\theta_{a} /\left(2 \sigma_{1}\right)}{\lambda k /(2 \alpha)+\theta_{a} \theta_{b} \lambda /\left(2 \sigma_{1}\right)+\theta_{a} k /\left(4 \alpha \sigma_{1}\right)}\right)^{-1}
$$

Here $\lambda=\left(\sigma_{2}-\sigma_{1}\right)^{-1}$ and the parameter $k$ is a geometric parameter of the interface defined by

$$
k=\Sigma_{j} \int_{\partial \mathbf{Y}^{j}}\left|y-r^{j}\right|^{2} d S
$$

where $\partial \mathbf{Y}^{j}$ is the surface of the $j$ th particle, $r^{j}=\left|\left(\partial \mathbf{Y}^{j}\right)\right|^{-1} \int_{\partial \mathbf{Y}^{j}} y d s$, and the sum is taken over all particles. The bound is easily seen to be monotonically decreasing in $k$. We apply Theorem 3.1 together with the bounds on $\sigma_{-}{ }^{e}$ to obtain the following theorem.

THEOREM 5.1 (bounds on effective conductivity for composites with highly conducting interface). The effective conductivity $\sigma_{+}{ }^{e}\left(\sigma_{1}, \sigma_{2}, \alpha\right)$ for an isotropic suspension of particles of conductivity $\sigma_{1}$ in a matrix of $\sigma_{2}$, with tangential conductivity $\alpha$, fixed specific arclength $s$, matrix phase tortuosity $\tau$, interface parameter $k$, and particle area fraction $\theta_{a}$, satisfies

$$
L^{+}\left(\sigma_{1}, \sigma_{2}, \alpha, k, \theta_{a}\right) \leq \sigma_{+}{ }^{e}\left(\sigma_{1}, \sigma_{2}, \alpha\right) \leq \mathcal{U}^{+}\left(\sigma_{1}, \sigma_{2}, \alpha, \tau, \theta_{a}\right),
$$

where

$$
\begin{gathered}
L^{+}\left(\sigma_{1}, \sigma_{2}, \alpha, k, \theta_{a}\right)=\left(\mathcal{U}\left(\sigma_{1}^{-1}, \sigma_{2}^{-1}, \alpha^{-1}, k, \theta_{a}\right)\right)^{-1} \\
=\sigma_{1}+\frac{\theta_{b} k /(2 \alpha)+\theta_{a}^{2} \lambda+\theta_{a} /\left(2 \sigma_{1}\right)}{\lambda k /(2 \alpha)+\theta_{a} \theta_{b} \lambda /\left(2 \sigma_{1}\right)+\theta_{a} k /\left(4 \alpha \sigma_{1}\right)}
\end{gathered}
$$


and

$$
\begin{gathered}
\mathcal{U}^{+}\left(\sigma_{1}, \sigma_{2}, \alpha, \tau, \theta_{a}\right)=\left(L\left(\sigma_{1}{ }^{-1}, \sigma_{2}{ }^{-1}, \alpha^{-1}, m, \theta_{a}\right)\right)^{-1} \\
=\sigma_{2}\left(1-\frac{1}{\left(1-\theta_{b} / \tau\right)^{-1}+\left(\sigma_{2}{ }^{-1} \theta_{a} c\right)^{-1}}\right)^{-1} .
\end{gathered}
$$

REMARK 5.2. Elementary bounds show that $0 \leq m \leq \theta_{b}$; hence the tortuosity satisfies $1 \leq \tau \leq \infty$.

Analysis shows that for fixed tortuosity, area fraction, and $\alpha \geq 0, \mathcal{U}^{+}\left(\sigma_{1}, \sigma_{2}, \alpha, \tau, \theta_{a}\right)$ is monotone increasing in $\alpha$ and

$$
\begin{gathered}
\sigma_{+}{ }^{e}\left(\sigma_{1}, \sigma_{2}, \alpha\right) \leq \mathcal{U}^{+}\left(\sigma_{1}, \sigma_{2}, \alpha, \tau, \theta_{a}\right) \leq \mathcal{U}^{+}\left(\sigma_{1}, \sigma_{2}, \infty, \tau, \theta_{a}\right) \\
=\sigma_{2} \tau / \theta_{b} .
\end{gathered}
$$

Remark 5.3. Passing to the limit $\alpha=\infty$ in (2.8) shows that $\sigma_{+}{ }^{e}=\sigma_{2} \tau / \theta_{b}$. In this way we see that the upper bound is optimal in this limit. (The passage to the limit $\alpha=\infty$ is justified using standard epi-convergence arguments; cf. Attouch [1].)

On the other hand, the bound $\mathcal{U}^{+}\left(\sigma_{1}, \sigma_{2}, \alpha, \tau, \theta_{a}\right)$ is found to be monotone increasing in $\tau$ for fixed area fraction and $\alpha \geq 0$. Calculation shows that

$$
\begin{gathered}
\sigma_{+}{ }^{e}\left(\sigma_{1}, \sigma_{2}, \alpha\right) \leq \mathcal{U}^{+}\left(\sigma_{1}, \sigma_{2}, \alpha, \tau, \theta_{a}\right) \leq \mathcal{U}^{+}\left(\sigma_{1}, \sigma_{2}, \alpha, \infty, \theta_{a}\right) \\
=\theta_{a} \sigma_{1}+\theta_{b} \sigma_{2}+s \frac{\alpha}{2} .
\end{gathered}
$$

Here $\mathcal{U}^{+}\left(\sigma_{1}, \sigma_{2}, \alpha, \infty, \theta_{a}\right)$ is the analogue of the Wiener upper bound for perfectly bonded conductors. For fixed values of $k$ one easily sees that the lower bound $L^{+}\left(\sigma_{1}, \sigma_{2}, \alpha, k, \theta_{a}\right)$ is monotone decreasing in $\alpha$. Passing to the $\alpha=0$ limit we have

$$
\begin{aligned}
\sigma_{+}{ }^{e}\left(\sigma_{1}, \sigma_{2}, \alpha\right) & \geq L^{+}\left(\sigma_{1}, \sigma_{2}, \alpha, k, \theta_{a}\right) \geq L^{+}\left(\sigma_{1}, \sigma_{2}, 0, k, \theta_{a}\right) \\
& =\sigma_{1}+\frac{\theta_{b}}{\frac{1}{\sigma_{2}-\sigma_{1}}+\frac{\theta_{a}}{2 \sigma_{1}}} \equiv H S_{-} .
\end{aligned}
$$

Here $H S_{-}$is the Hashin-Shtrikman lower bound for perfectly bonded two-phase conductors [5].

6. Size effects, critical radius, and energy-minimizing polydisperse suspensions of disks. We consider suspensions of inclusions of conductivity $\sigma_{1}$ embedded in a matrix of higher conductivity $\sigma_{2}$. For fixed-component volume fractions we use the monotonicity of the bounds in the interfacial parameter $k$ and specific surface $s$ to identify a distinguished parameter $\mathcal{P}_{c r}=\alpha /\left(\sigma_{2}-\sigma_{1}\right)$. We shall show for monodisperse suspensions of disks that $\mathcal{P}_{c r}$ picks out the scale at which the effects of the interface balance the conductivity mismatch between component phases. The bounds will also serve as a tool for understanding the role of surface energy in problems of energy-minimizing arrangements of polydisperse suspensions of disks.

In this section we will consider only suspensions for which the associated effective tensor is isotropic.

We start by considering monodisperse suspensions of disks. We fix the area fraction of particles and state the following.

THEOREM 6.1. Given that the common radius of a monodisperse suspension of disks is $r$, then

$$
\begin{array}{ll}
\sigma_{+}{ }^{e}\left(\sigma_{1}, \sigma_{2}, \alpha\right)>\sigma_{2} & \text { for } r<\mathcal{P}_{c r}, \\
\sigma_{+}{ }^{e}\left(\sigma_{1}, \sigma_{2}, \alpha\right)<\sigma_{2} & \text { for } r>\mathcal{P}_{c r},
\end{array}
$$


and

$$
\sigma_{+}{ }^{e}\left(\sigma_{1}, \sigma_{2}, \alpha\right)=\sigma_{2} \quad \text { for } r=\mathcal{P}_{c r} .
$$

Proof. For monodisperse suspensions of common radius $r$ the geometric parameters $k$ and $s$ are given by

$$
k=2 \theta_{a} r, \quad s=2 \theta_{a} r^{-1} .
$$

When $r=\mathcal{P}_{c r}$ substitution of (6.4) into the upper bound $\mathcal{U}^{+}$gives

$$
\mathcal{U}^{+}=\sigma_{2} .
$$

For $r=\mathcal{P}_{c r}$ substitution of (6.4) into the lower bound $L^{+}$gives

$$
L^{+}=\sigma_{2},
$$

and (6.3) is proven. Inequalities (6.1) and (6.2) follow immediately from the monotonicity of the bounds in the geometric parameters.

Theorem 6.1 shows that the mismatch between matrix and particle conductivity is compensated by the highly conducting interface for particles with radius $\mathcal{P}_{c r}$. In the following section we will show that this phenomenon persists for any dispersion of particles of radius $\mathcal{P}_{c r}$.

For polydisperse suspensions of disks we define the mean radius $\langle r\rangle$ of the suspension by

$$
\langle r\rangle=\sum_{j=1}^{N} \frac{\left|\mathbf{Y}^{j}\right|}{\theta_{a}} a^{j},
$$

where $a^{j}$ is the radius of the $j$ th disk, $\mathbf{Y}^{j}$ is the region occupied by the $j$ th disk, $\left|\mathbf{Y}^{j}\right|$ is its area fraction, and $\theta_{a}$ is the total volume area occupied by the particles.

For polydisperse suspensions of spheres we state the following theorem.

THEOREM 6.2 (size effect theorem for polydisperse suspensions of disks). For polydisperse suspensions of disks of conductivity $\sigma_{1}$ and matrix of conductivity $\sigma_{2}$ with $\sigma_{1}<\sigma_{2}$ and particle area fraction $\theta_{a}$, if $\langle r\rangle \leq \mathcal{P}_{c r}$, then

$$
\sigma_{+}{ }^{e}\left(\sigma_{1}, \sigma_{2}, \alpha\right) \geq \sigma_{2} .
$$

One has equality in (6.8) only if

$$
\langle r\rangle=\mathcal{P}_{c r} .
$$

Proof. For polydisperse suspensions of disks, the parameter $k$ is given by

$$
k=2 \theta_{a}\langle r\rangle .
$$

Substitution of (6.10) into the lower bound (5.7) shows that the lower bound is strictly monotonically increasing as the mean radius tends to zero. For $\langle r\rangle=\mathcal{P}_{c r}$ one has

$$
L^{+}\left(\sigma_{1}, \sigma_{2}, \alpha, 2 \theta_{a}\langle r\rangle, \theta_{a}\right)=\sigma_{2},
$$

and the theorem follows. 
We now consider particulate suspensions with no assumption on particle shape or distribution other than that the resulting effective conductivity is isotropic. For this case we have the following theorem.

THEOREM 6.3. For suspensions of particles of conductivity $\sigma_{1}$ in a matrix of conductivity $\sigma_{2}$, with $\sigma_{2}>\sigma_{1}$ and the particle area fraction $\theta_{a}$ prescribed, if the specific arclength $s$ is bounded above by $2 \theta_{a} \mathcal{P}_{c r}^{-1}$, i.e.,

$$
s \leq 2 \theta_{a} \mathcal{P}_{c r}^{-1},
$$

then

$$
\sigma_{+}{ }^{e}\left(\sigma_{1}, \sigma_{2}, \alpha\right) \leq \sigma_{2} .
$$

Proof. For $s=2 \theta_{a} P_{c r}^{-1}$ it follows from (5.8) that the upper bound $\mathcal{U}^{+}=\sigma_{2}$. Moreover, since the upper bound is monotone increasing in $s$, one has that $\mathcal{U}^{+} \leq \sigma_{2}$ for $s \leq 2 \theta_{a} \mathcal{P}_{c r}^{-1}$, and the theorem follows.

It is evident from its definition, that effective conductivity is equivalent to the energy dissipated inside a two-phase conductor; see (2.7). In this regard, we see that Theorems 6.1, 6.2, and 6.3 are energy-dissipation theorems for a system with bulk and interfacial energy. In what follows we consider only isotropic polydisperse suspensions of disks. We fix the area fraction of disks and examine the role of surface energy in selecting a suspension with minimum isotropic effective conductivity $\sigma_{+}{ }^{e}\left(\sigma_{1}, \sigma_{2}, \alpha\right)$. In what follows we present a necessary condition on the size distribution of the disks appearing in the optimal suspension. We remark that it is not known if a minimal suspension exists. Instead, as with many problems, there may be no minimum but only a minimizing sequence of suspensions. For this case, the same necessary condition will hold in the appropriate sense for minimizing sequences of suspensions.

To fix ideas we suppose that the area fraction of disks satisfies the inequality

$$
\theta_{a}<\pi / 4
$$

That is, that the area fraction is less than a circle of radius $1 / 2$ inscribed within the unit cell. Moreover, we restrict the parameters $\sigma_{1}, \sigma_{2}$, and $\alpha$ so that $\mathcal{P}_{c r}$ satisfies the constraint

$$
\pi \mathcal{P}_{c r}^{2}<\theta_{a}
$$

The above states that we consider only cases where the area of a single disk of critical radius $\left(\mathcal{P}_{c r}\right)$ is strictly less than the area fraction occupied by the suspension. We have the following theorem characterizing the optimal polydisperse suspension minimizing the effective conductivity.

THEOREM 6.4 (optimal design necessary condition). Given $\theta_{a}$ and $\sigma_{1}, \sigma_{2}$, and $\alpha$ satisfying the constraints (6.14) and (6.15), the mean radius of the optimal distribution of spheres minimizing $\sigma_{+}{ }^{e}\left(\sigma_{1}, \sigma_{2}, \alpha\right)$ is greater than $\mathcal{P}_{c r}$.

Proof. From Theorem 6.2 we know that if the mean radius lies below $\mathcal{P}_{c r}$, then $\sigma_{+}{ }^{e} \geq \sigma_{2}$. So to establish the theorem we construct a polydisperse suspension of spheres with mean radius greater than $\mathcal{P}_{c r}$ with effective conductivity less than $\sigma_{2}$. The construction is simple in view of (6.14) and (6.15). Indeed, take a suspension consisting of a single sphere centered in the period cell of radius $r$ such that

$$
\pi r^{2}=\theta_{a}
$$


Then for this suspension,

$$
s /\left(2 \theta_{a}\right)=r^{-1} .
$$

From (6.14) and (6.15) we see that

$$
r^{-1}<\mathcal{P}_{c r}^{-1}
$$

therefore,

$$
s<2 \theta_{2} \mathcal{P}_{c r}^{-1} .
$$

We conclude from (6.19) and Theorem 6.3 that $\sigma_{+}{ }^{e}<\sigma_{2}$, and the theorem is established.

This theorem shows that the scale of the heterogeneity plays a role in the extremal transport properties of a polydisperse distribution of disks. This is in striking contrast to optimal layout problems with perfect transmission between phases where scale plays no role in the optimal design; see Lurie and Cherkaev [9] and Murat and Tartar [11]. We observe that these effects are a direct result of the surface energy dissipated by composites with highly conducting interfaces.

7. Cloaking of inclusions. In the previous section we used bounds to find a critical radius for monodisperse suspensions with isotropic effective conductivity tensor. At this radius the effective conductivity of the suspension is seen to equal that of the matrix. Here we show that this phenomenon persists even for anisotropic suspensions in arbitrary domains. In doing so we obtain a fundamental result concerning the behavior of electric fields inside suspensions at critical radius.

We consider two- and three-dimensional monodisperse suspensions of disks (spheres) of conductivity $\sigma_{1}$ embedded in a matrix of $\sigma_{2}$ with $\sigma_{2}>\sigma_{1}$. The suspension is contained inside a bounded open set in $\Omega$ in $\mathbb{R}^{d}(d=2,3)$ with Lipschitz boundary. We require that none of the inclusions touch each other or intersect the boundary of $\Omega$. We suppose that the suspension consists of $N$ spheres of equal radius. The boundary of the $m$ th sphere is denoted by $\partial \mathbf{Y}^{m}$, and the union of all sphere boundaries is written as $\Gamma$. The electric potential $\varphi$ inside the suspension satisfies

$$
\begin{gathered}
\Delta \varphi=0 \quad \text { in each phase, } \\
{[\varphi]_{2}^{1}=0 \quad \text { on } \partial \mathbf{Y}^{m}, m=1, \ldots N,} \\
{[\sigma(x) \nabla \varphi \cdot \mathbf{n}]_{2}^{1}=\alpha \delta_{i}^{2} \varphi \quad \text { on } \partial \mathbf{Y}^{m}, m=1, \ldots, N .}
\end{gathered}
$$

Here $\mathbf{n}$ is the unit outer normal to $\partial \mathbf{Y}^{m}$ and $[\cdot]_{2}^{1}$ denotes the jump in a quantity across the two-phase interface. We inject a uniform current into boundary $\partial \Omega$, i.e.,

$$
\sigma_{2} \partial_{n} \varphi=\sigma_{2} \mathbf{E} \cdot \mathbf{n} \quad \text { on } \delta \Omega,
$$

where $\mathbf{E}$ is a constant electric field in $\mathbb{R}^{d}$. Integration by parts shows that the total energy dissipation rate $W$ inside the composite is given by

$$
W=\int_{\partial \Omega} \varphi \sigma_{2} \partial_{n} \varphi d S=\int_{\Omega} \sigma(x)|\nabla \varphi|^{2} d x+\alpha \int_{\Gamma}|\delta \varphi|^{2} d S .
$$

For $d=2$ we have as in the previous section

$$
\mathcal{P}_{c r}=\alpha /\left(\sigma_{2}-\sigma_{1}\right),
$$


and for $d=3$ we introduce $\tilde{\mathcal{P}}_{c r}$, defined by

$$
\tilde{\mathcal{P}}_{c r}=2 \alpha /\left(\sigma_{2}-\sigma_{1}\right) .
$$

We consider values for the conductivities $\sigma_{1}, \sigma_{2}, \alpha$ for which monodisperse suspensions of nonintersecting disks (spheres) of radius $\mathcal{P}_{c r}\left(\tilde{\mathcal{P}}_{c r}\right)$ are contained in the region $\Omega$ and state the following theorem.

THEOREM 7.1. The electric field inside a monodisperse suspension of disks (spheres) of radius $\mathcal{P}_{c r}\left(\tilde{\mathcal{P}}_{c r}\right)$ contained inside $\Omega$ is uniform. The associated electric potential $\varphi$ is given by

$$
\varphi=\mathbf{E} \cdot x \quad \text { in } \Omega
$$

and the current $\mathbf{j}$ inside $\Omega$ is given by

$$
\mathbf{j}=\left\{\begin{array}{l}
\sigma_{1} \mathbf{E} \quad \text { inside each sphere } \\
\sigma_{2} \mathbf{E} \quad \text { in the matrix }
\end{array}\right.
$$

where $\varphi$ and $\mathbf{j}$ given by (7.8) and (7.9) are independent of the number and location of the spheres.

We see that the potential in the suspension is the same as if there were no particles of conductivity $\sigma_{1}$. A straightforward calculation shows the following.

COROLlaRY 7.2. The total energy dissipation rate $W$ for a monodisperse suspension of disks (spheres) of critical radius $\mathcal{P}_{c r}\left(\tilde{\mathcal{P}}_{c r}\right)$ is given by

$$
W=\left(\sigma_{2} \mathbf{E} \cdot \mathbf{E}\right)|\Omega|
$$

independently of the number and location of the spheres.

REMARK 7.3. We observe that the energy dissipation rate given by (7.10) is identical to the dissipation rate obtained if the region were composed only of $\sigma_{2}$ conductor.

On the other hand, suppose that we are given a suspension of $N$ spheres of common radius $r$ contained in $\Omega \subset \mathbb{R}^{3}$, and we are free to choose the particle, matrix, and tangential conductivities. Then we have the following corollary.

COROLLARY 7.4 (cloaking of spheres). Given a monodisperse suspension of $N$ spheres of radius $r$ such that the spheres are not touching or come into contact with the boundary. Then if the tangential conductivity $\alpha$ and conductance mismatch $\sigma_{2}-\sigma_{1}$ are chosen such that $\sigma_{2}-\sigma_{1}>0$ and

$$
\frac{2 \alpha}{\sigma_{2}-\sigma_{1}}=r
$$

then the electric field inside the composite is uniform and the associated potential and current are given by (7.7) and (7.8).

REMARK 7.5. For two-dimensional monodisperse suspensions of disks the conclusion of Corollary 7.4 is obtained, provided that $\sigma_{2}-\sigma_{1}>0$ and

$$
\frac{\alpha}{\sigma_{2}-\sigma_{1}}=r .
$$

REMARK 7.6. If both the common radii $r$ of the spheres and the conductivities $\sigma_{2}$ and $\sigma_{1}$ with $\sigma_{2}>\sigma_{1}$ are prescribed, then Corollary 7.4 shows one how to choose the appropriate tangential conductivity a to render the spheres undetectable.

We now give the proof of Theorem 7.1. To fix ideas we treat the three-dimensional problem noting that the two-dimensional solution follows the same lines. To establish 
the theorem we consider a suspension of $N$ spheres of common radius $r$ with centers denoted by $r^{m}$. We suppose that the potential $\varphi$ is of the form

$$
\varphi= \begin{cases}\mathbf{E} \cdot x & \text { in the matrix } \\ \zeta \cdot x+c_{j} & \text { in the } j \text { th particle }\end{cases}
$$

where $c_{j}$ is a constant and $\zeta$ is a constant vector in $\mathbb{R}^{3}$. We show that for $r=\tilde{\mathcal{P}}_{c r}$ there exists a potential of the form (7.13) and it is given by (7.8).

To start, we see that the function given by (7.13) satisfies (7.1) and (7.4). Continuity of the potential implies the compatibility condition

$$
\zeta-\mathbf{E}=\lambda \mathbf{n} \quad \text { on } \partial \mathbf{Y}^{m},
$$

where $\lambda$ is a scalar to be determined.

Since $\varphi$ is continuous, we have

$$
\frac{1}{|\Omega|} \int_{\Omega} \nabla \varphi d x=\mathbf{E}=\theta_{a} \zeta+\theta_{b} \mathbf{E}
$$

It is evident from (7.15) and $\theta_{a}=1-\theta_{b}$ that $\zeta=\mathbf{E}$ and (7.14) holds with $\lambda=0$. Thus we find that $\varphi=\mathbf{E} \cdot x$ in $\Omega$. On the other hand, (7.3) becomes

$$
\left(\sigma_{1}-\sigma_{2}\right) \mathbf{E} \cdot \mathbf{n}=\alpha \delta_{i}^{2}(\mathbf{E} \cdot x) \quad \text { on } \partial \mathbf{Y}^{m}
$$

and must be satisfied. This equation provides the extra condition necessary to determine the common radius of the suspension.

Computation of $\delta_{i}^{2}(\mathbf{E} \cdot x)$ on the $m$ th sphere and rewriting (7.16) give

$$
\left(\sigma_{1}-\sigma_{2}\right) \mathbf{E} \cdot \mathbf{n}=-\frac{\alpha}{r} 2 \mathbf{E} \cdot \mathbf{n} \quad \text { on } \partial \mathbf{Y}^{m} .
$$

It is easily seen that (7.17) holds, provided that $r=\tilde{\mathbf{P}}_{c r}$, and the theorem is proven.

Acknowledgments. The author wishes to thank Bogdan Vernescu for stimulating conversations.

\section{REFERENCES}

[1] H. Аттоuch, Variational Convergence for Functions and Operators, Pitman, Boston, 1984.

[2] F. A. L. Dullien, Porous Media Fluid Transport and Pore Structure, Academic Press, New York, 1979.

[3] K. W. Garett and H. M. Rosenberg, The thermal conductivity of epoxy-resin/powder composite materials, Journal of Physics D: Applied Physics, 7 (1974), pp. 1247-1258.

[4] J. B. Keller, A theorem on the conductivity of a composite medium, J. Math. Phys. 5 (1964), pp. 548-549.

[5] Z. Hashin And S. Shtrikman, A variational approach to the theory of effective magnetic permeability of multiphase materials, J. Appl. Phys., 33 (1962), pp. 3125-3131.

[6] R. Holm, Electric Contacts, Springer-Verlag, Berlin, 1967.

[7] R. Lipton, Heat conduction in fine scale mixtures with interfacial barrier resistance, SIAM J. Applied Math., 57 (1997), to appear.

[8] R. Lipton and B. Vernescu, Composites with imperfect interface, Proc. Roy. Soc. London Ser. A, 452 (1996), pp. 325-358.

[9] K. A. Lurie And A. V. Cherkaev, Effective characteristics of composite materials and the optimal design of structural elements, Adv. Mech., 9 (1986), pp. 3-81.

[10] K. S. Mendelson, J. Appl. Phys., 46 (1975), p. 917. 
[11] F. Murat And L. TARTAR, Calcul des Variations et Homogénéisation: Théorie et Applications en Physique, Coll. de la Dir. des Etudes et Reserches de Electr. del France, Eyrolles, Paris, 1985, pp. 319-370.

[12] J. F. Nye, Physical Properties of Crystals: Their Representation by Tensors and Matrices, Clarendon Press, Oxford, 1967.

[13] H. Pham Huy and E. Sanchez-Palencia, Phénomènes de à travers des couches minces de conductivité élevée, J. Math. Anal. Appl., 47 (1974), pp. 284-309.

[14] E. Sanchez-Palencia, Non-Homogeneous Media and Vibration Theory, Lecture Notes in Phys., Springer-Verlag, Berlin, 1980.

[15] S. TORQuATO AND M. D. Rintoul, Effects of the interface on the properties of composite media, Phys. Rev. Lett., 75 (1995), pp. 4067-4070. 